\title{
Drug-eluting balloon catheters for lower limb peripheral arterial disease: the evidence to date
}

\author{
This article was published in the following Dove Press journal: \\ Vascular Health and Risk Management \\ 12 May 2016 \\ Number of times this article has been viewed
}

\section{Mohamed Barkat' \\ Francesco Torella' George A Antoniou ${ }^{2}$ \\ 'Liverpool Vascular and Endovascular Service, Royal Liverpool University Hospital, Liverpool, ${ }^{2}$ Department of Vascular and Endovascular Surgery, The Royal Oldham Hospital, Pennine Acute Hospitals NHS Trust, Manchester, UK}

\begin{abstract}
A significant proportion of patients with severe lower limb peripheral arterial disease require revascularization. Over the past decade, an endovascular-first approach even for complex disease has gained widespread use among vascular specialists. An important limitation of percutaneous transluminal balloon angioplasty or stenting remains the occurrence of restenosis. Drugcoated balloons have emerged as an exciting technology developed to overcome the limitations of standard balloon angioplasty and stenting. Drug-eluting devices inhibit neointimal growth of vascular smooth muscle cells with the potential of preventing restenosis. This review provides a synopsis of the up-to-date evidence on the role of drug-coated balloons in the treatment of lower limb peripheral arterial disease. Bibliographic searches were conducted using MEDLINE, EMBASE, and the Cochrane Library electronic database. Eleven randomized clinical trials, two systematic reviews, and a published registry providing the best available evidence were identified. Current evidence suggests that angioplasty with drug-coated balloon is reliable, safe, and efficient in increasing patency rates and reducing target lesion revascularization and restenosis. However, it remains unknown whether these improved results can translate into beneficial clinical outcomes, as current randomized clinical trials have failed to demonstrate a significant benefit in limb salvage and mortality. Further randomized trials focusing on clinical and functional outcomes of drug-eluting balloons and on cost versus clinical benefit are required.
\end{abstract}

Keywords: drug-eluting balloon, drug-coated balloon, angioplasty, peripheral arterial disease

\section{Introduction}

Atherosclerosis is a systemic disease of the large- and medium-sized arteries causing luminal narrowing (focal or diffuse) as a result of the accumulation of lipid and fibrous material between the intimal and medial layers of the vessel. ${ }^{1}$ Atherosclerosis of the noncardiac vessels is defined as peripheral artery disease (PAD). PAD can present clinically as intermittent claudication (IC), which can severely impair lifestyle. More severe disease may present as critical limb ischemia (CLI) with rest pain, ulceration, or gangrene in the lower extremities. The worldwide prevalence of PAD is between $3 \%$ and $12 \%{ }^{2}$ In Europe and North America, an estimated 27 million individuals are affected, with $\sim 413,000$ inpatient admissions annually attributed to PAD. ${ }^{2}$

The European Society of Cardiology Guidelines, published in 2011, recommended an endovascular-first strategy in all femoral-popliteal TASC A-C and infrapopliteal lesions, when revascularization is indicated. ${ }^{3}$ The low morbidity and mortality of endovascular techniques, such as percutaneous transluminal angioplasty (PTA) and stenting, make it the preferred choice of treatment in diseases such as stenosis and
Correspondence: George A Antoniou Surgical Offices, Top Floor, Phase I, The Royal Oldham Hospital, Rochdale Road, Oldham OLI 2JH, UK

$\mathrm{Tel}+4475$ 07। I 246 I

Email antoniou.ga@hotmail.com (c) (1) (5) 2016 Barkat et al. This work is published and licensed by Dove Medical Press Limited. The full terms of this license are available at https://www.dovepress.com/terms. cC. you hereby accept the Terms. Non-commercial uses of the work are permitted without any further permission from Dove Medical Press Limited, provided the work is properly attributed. For permission for commercial use of this work, please see paragraphs 4.2 and 5 of our Terms (https://www.dovepress.com/terms.php). 
occlusions. ${ }^{3}$ However, the main drawback of this strategy is its considerable restenosis rate, ranging from $40 \%$ to $60 \%$ at 12 months. Restenosis is usually caused by neointimal hyperplasia and may lead to recurrent symptoms. ${ }^{4}$

Drug-eluting devices, which inhibit neointimal growth of vascular smooth muscle cells, may prevent restenosis. In recent years, drug-eluting balloons (DEBs) have emerged as an exciting technology developed to overcome the limitations of drug-eluting stents (DESs), such as stent thrombosis and dependency on prolonged dual antiplatelet therapy, and may prove efficacious in complex subsets such as small vessels and diffuse lesions, where stent results are suboptimal. ${ }^{5}$ In addition, DEBs have the potential for higher drug tissue bioavailability due to the higher drug surface area presented to the vessel wall compared with DESs. ${ }^{6}$

DEBs available today utilize paclitaxel in combination with different excipients. Paclitaxel is used as an antiproliferative agent for the prevention of restenosis. It is highly lipophilic, which promotes a rapid cellular uptake. It exerts potent and sustained inhibitory effects on smooth muscle cell proliferation and migration known to occur during the restenosis process in arteries, even after single-dose application. ${ }^{7}$ Excipient is a hydrophilic spacer (urea or polymers), which enables rapid drug transfer to the medial and adventitial layer of the arterial wall. ${ }^{8}$

Recently, several clinical studies and randomized control trials (RCTs) have been performed to assess the efficacy and safety of DEBs in the treatment of PAD. The purpose of this review is to provide up-to-date evidence on the role of DEBs in the treatment of femoral-popliteal and infrapopliteal PAD.

\section{Methodology}

\section{Search strategy}

To provide up-to-date evidence, bibliographic searches were conducted to identify all publications related to DEB angioplasty for the treatment of lower limb PAD. MEDLINE, EMBASE, and the Cochrane Library electronic database were searched for articles published between 1980 and July 2015. The search terms were "angioplasty", "drug eluting", "drug coated balloon", "paclitaxel”, "drug eluting balloon angioplasty", "peripheral arterial disease", "peripheral vascular disease", "infra-inguinal", "superficial femoral artery", "popliteal", "tibial", and "peroneal".

\section{Study selection}

Abstracts found in literature searches were independently screened for potential studies of interest. Studies were limited to the English language and adult population of any age group. Inclusion criteria were all RCTs, systematic reviews, registries, and large cohort studies evaluating the role of DEB angioplasty in the treatment of de novo femoral-popliteal and infrapopliteal lesions. We focused on the following primary end points: binary restenosis, late lumen loss (LLL), target lesion revascularization (TLR), mortality, and major amputation rate.

\section{Search outcome}

The primary search for DEB angioplasty in PAD returned 190 citations. Twenty-four relevant publications met the inclusion criteria for this review. Fourteen of these articles provided the best up-to-date evidence on DEBs in PAD. Seven RCTs and two meta-analyses reported on DEBs in femoral-popliteal disease. Three RCTs and one registry reported on infrapopliteal disease, and another RCT reported on DEB intervention in both femoral-popliteal and infrapopliteal lesions.

\section{Study quality assessment}

The Cochrane collaboration's tool was applied to assess the risk of bias of RCTs. ${ }^{9}$ The Grades of Recommendation Assessment, Development, and Evaluation methodology was used to rate our confidence in each reported outcome as high, moderate, low, or very low on the basis of different domains. ${ }^{10}$

\section{Results}

\section{Femoral-popliteal disease}

Since 2008, eight RCTs ${ }^{11-18}$ and two meta-analyses ${ }^{19,20}$ have demonstrated favorable technical outcomes with DEBs compared with plain balloon angioplasty in the treatment of femoral-popliteal atherosclerotic disease, as indicated by LLL, restenosis rate, and freedom from TLR. Herein, we present the most important trials, based on the methodological and risk of bias assessment, the sample size, and the length of follow-up. A detailed description of all selected studies is outlined in Table 1. The LEVANT $2^{11}$ and IN.PACT $\mathrm{SFA}^{12}$ are the most recent international multicenter RCTs with the largest number of enrolled patients (331 and 476, respectively).

The LEVANT 2 pivotal Investigational Device Exemption trial is a prospective, multicenter (42 in US and 12 in European Union), single-blind, randomized (2:1) clinical trial comparing Lutonix DEB to standard PTA for the treatment of occlusive disease in native femoral-popliteal arteries. The primary patency at 1 year was $65.2 \%$ for the DEB group, which was superior to that of conventional PTA $(52.6 \%$; 


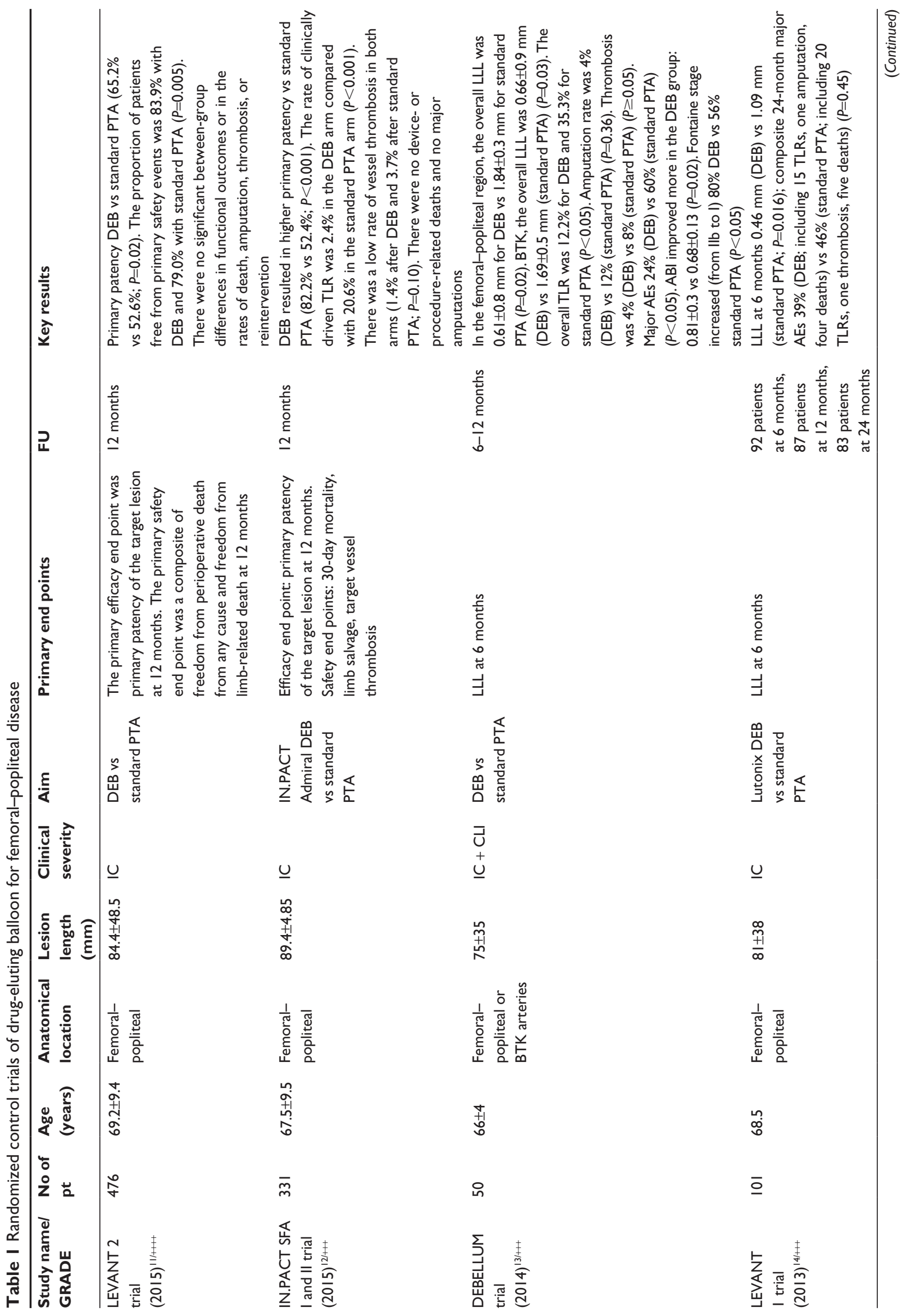




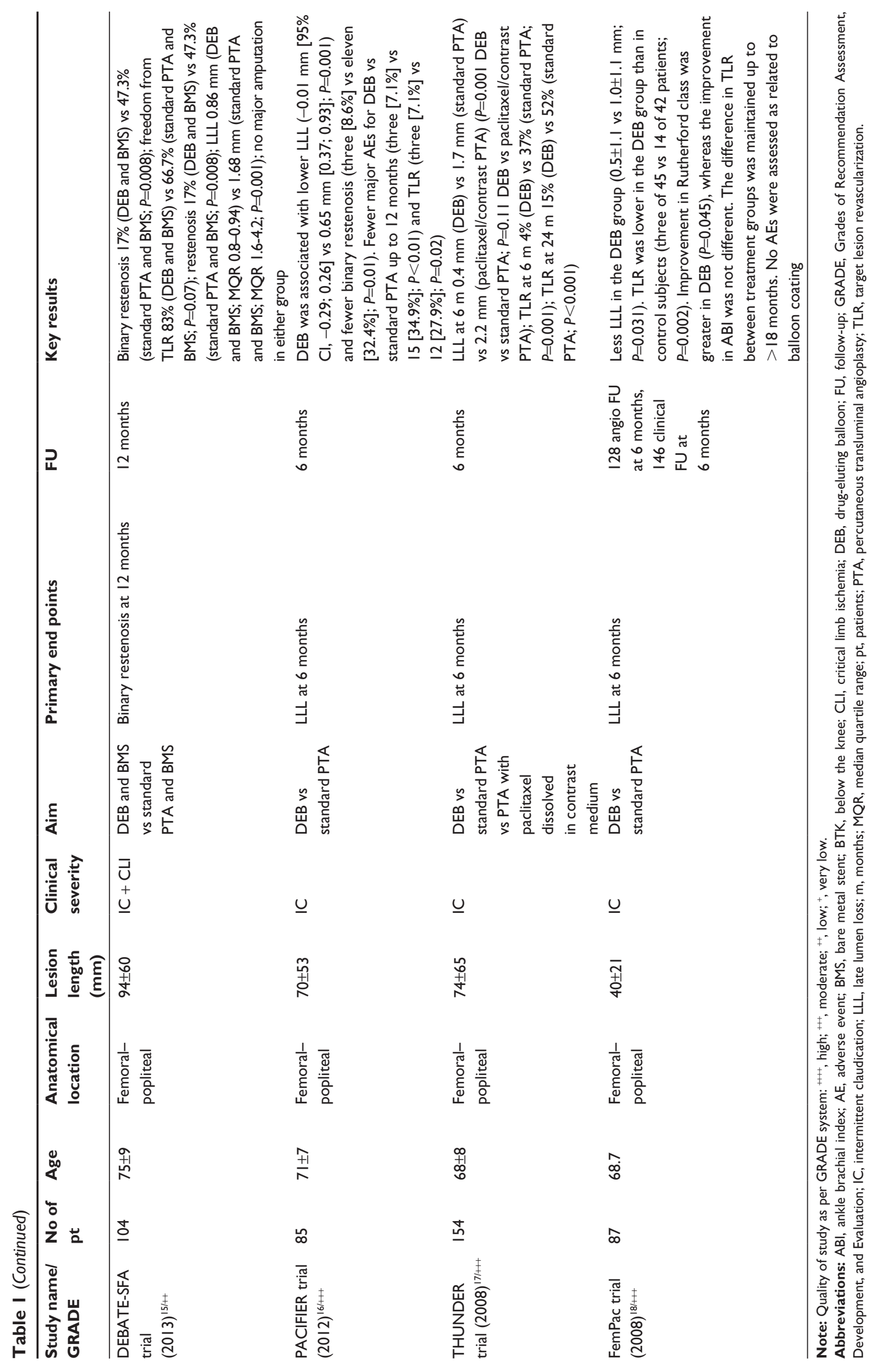


$P=0.015)$. Freedom from clinically driven TLR in the DEB group was $87.7 \%$ compared to $83.2 \%$ in the control group $(P=0.208)$. The absence of a significant difference might be due to the fact that $50 \%$ restenosis might not result in any significant clinical symptoms. The proportion of patients free from primary safety end points (freedom from perioperative death from any cause and freedom from limb-related death at 12 months) was $83.9 \%$ with DEBs and $79.0 \%$ with standard balloon angioplasty ( $P=0.005$ for noninferiority).

The IN.PACT SFA I and II are prospective, multicenter RCTs that enrolled 331 patients in 44 US and 13 European centers. Patients were randomized $2: 1$ to treatment with either the IN.PACT Admiral DEB or standard PTA. Clinically driven TLR rates were significantly lower with the DEBs as compared with those achieved with standard PTA $(2.4 \%$ vs $20.6 \%, P=0.001)$. Similarly, the primary patency rate at 12 months achieved with IN.PACT Admiral balloons was $82.2 \%$, while the primary patency achieved with standard PTA was $52.5 \%(P=0.001)$. In terms of safety end points, in both trials there were no procedure-related complications or major amputations reported. However, none of the trials involved patients with CLI. In addition, the mean lesion length was $63 \mathrm{~mm}$ in the LEVANT 2 trial.

A meta-analysis by Cassese et al, ${ }^{19}$ comparing DEB angioplasty versus standard PTA for femoral-popliteal disease in 381 patients with a mean follow-up of 10.3 months, demonstrated superior results with DEB angioplasty, as indicated by TLR ( $12.2 \%$ vs $27.7 \%$; odds ratio, $0.22 ; P=0.00001$ ), angiographic restenosis ( $18.7 \%$ vs $45.5 \%$; odds ratio, 0.26 ; $P=0.00001$ ), and LLL (mean weighted difference, $0.75 \mathrm{~mm}$; $P=0.00001)$. There was no mortality difference between the two groups. A more recent meta-analysis by Baerlocher et $\mathrm{al}^{20}$ also demonstrated superior technical outcomes of DEBs, as indicated by LLL, restenosis, and TLR, compared to PTA, but no difference in clinical outcome parameters such as amputation and mortality.

\section{Infrapopliteal disease}

Following encouraging results with DEBs in the femoralpopliteal segment, studies and clinical trials were conducted to assess the efficacy and safety of DEBs in below the knee (BTK) arterial disease (Table 2). First published data obtained from Schmidt et al, ${ }^{21}$ who conducted a prospective cohort study reporting on 104 consecutive patients (109 limbs) treated for CLI (82.6\%) or severe IC (17.4\%) due to BTK arterial disease with DEBs. Mean lesion length was $176 \pm 88 \mathrm{~mm}$. Angiography performed at 3 months to assess 84 of the treated arteries showed a restenosis rate of $27.4 \%$
(19.1\% had restenosis of $>50 \%$ and $8.3 \%$ were totally occluded). Restenosis usually occurred focally. During a follow-up period of $378 \pm 65$ days, one patient was lost and 17 died. Of the 91 limbs remaining in the analysis, clinical improvement was present in $83(91.2 \%)$. Complete wound healing occurred in $74.2 \%$, whereas major amputation occurred in four patients, resulting in a limb salvage rate of 95.6\% for patients with CLI.

Since 2013, four RCTs $\mathrm{s}^{13,22-24}$ have examined the efficacy of DEBs in BTK atherosclerotic disease. In the DEBELLUM trial, ${ }^{13} 50$ consecutive patients with 122 lesions in the femoral-popliteal and/or infrapopliteal arteries were randomized to DEBs or standard PTA. The preliminary 1-year results on the BTK lesions confirmed a better outcome with DEBs over standard PTA in terms of LLL $(0.66 \pm 0.9 \mathrm{~mm}$ DEB vs $1.69 \pm 1.5 \mathrm{~mm}$ PTA; $P<0.05)$, TLR $(15.3 \%$ DEB vs $47.0 \%$ PTA; $P<0.05)$, and primary patency $(84.6 \%$ DEB vs $41.1 \%$ PTA; $P<0.05)$. However, major adverse events (defined as major or minor amputation, thrombosis, or death) did not differ significantly between DEBs and standard PTA presumably because of the limited number of lesions and patients treated.

The DEBATE-BTK trial ${ }^{23}$ investigated the efficacy of a paclitaxel DEB for the reduction of restenosis in diabetic patients with CLI. Binary restenosis, assessed by angiography in $>90 \%$ of patients, occurred in 20 of $74(27 \%)$ lesions in the DEB group versus 55 of 74 (74\%) lesions in the standard PTA group $(P<0.001)$, TLR in $12(18 \%)$ versus $29(43 \%)(P=0.002)$, and target vessel occlusion in $12(17 \%)$ versus $41(55 \%)(P<0.001)$. There was one major amputation, which occurred in the standard PTA group $(P=0.9)$.

In the IDEAS trial, ${ }^{24} 50$ patients were randomized to infrapopliteal DEB angioplasty (25 arteries in 25 limbs) or primary DES placement (30 arteries in 27 limbs). The binary restenosis rate was significantly lower in DES $(28 \%$ vs $57.9 \% ; P=0.0457)$. There were no significant differences in TLR (7.7\% in DES vs $13.6 \%$ in DEB; $P=0.65)$. At 6 months, five patients died (two in DEB vs three in DES; $P=1.00$ ) and three suffered a major amputation (one in DEB vs two in DES; $P=1.00)$.

In the IN.PACT DEEP trial, ${ }^{22} 358$ patients with CLI were randomized 2:1 to IN.PACT Amphirion DEB angioplasty or standard PTA at 13 European sites. After 12 months, the decision was made to recall the IN.PACT Amphirion DEB based on a trend toward a higher rate of major amputation in the DEB arm $(8.8 \%$ vs $3.6 \% ; P=0.08)$ and no significant benefit for the efficacy end points of clinically driven TLR (11.9\% vs $13.5 \% ; P=0.682)$, LLL $(0.605 \pm 0.775 \mathrm{~mm}$ vs 


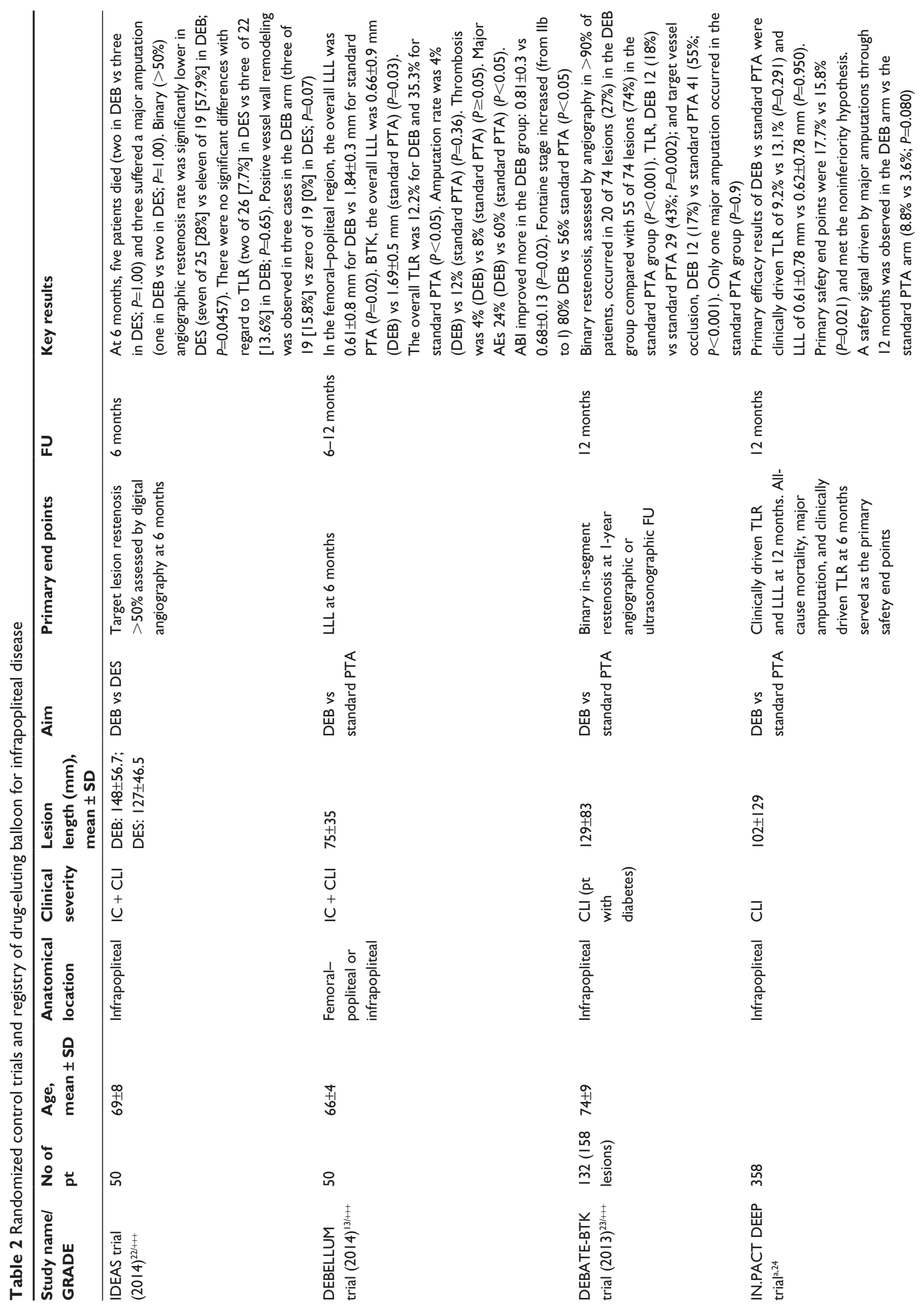



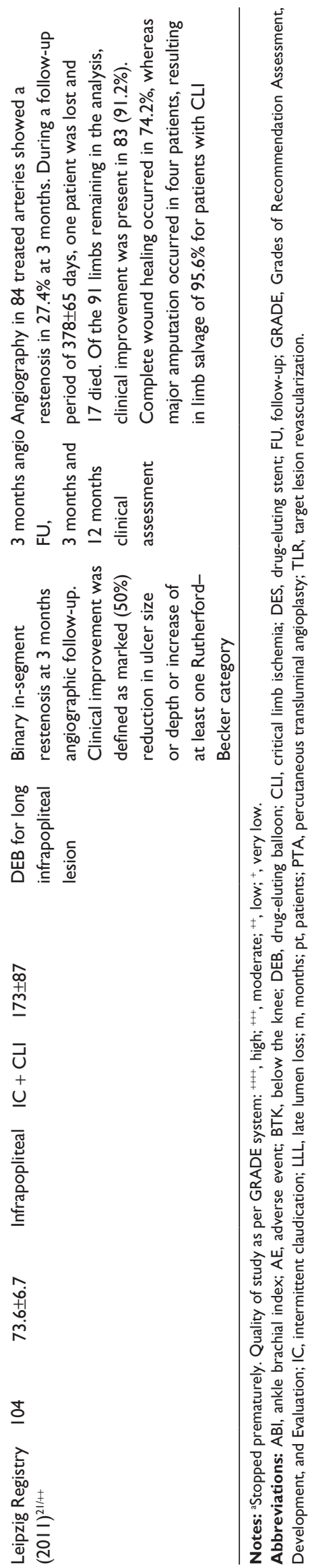

$0.616 \pm 0.781 \mathrm{~mm} ; P=0.950)$, and binary restenosis ( $41 \%$ vs $35.5 \% ; P=0.609$ ).

\section{Risk of bias assessment}

The results of the risk of bias of the RCTs are outlined in Figures 1 and 2. All RCTs included in this review demonstrated low selection bias as a random sequence generation was applied. It terms of performance bias, it was not possible to blind the interventionist who performed the procedure (DEB angioplasty or standard PTA) in all the trials, which led to high performance bias. However, patients were blinded to the treatment they received. In the DEBATE-SFA ${ }^{15}$ and $\mathrm{BKA}^{23}$ trials, an external data adjudication committee or core lab was lacking due to the financial constraints of running an independent trial, which resulted in the judgment of a high detection bias. In the FemPac ${ }^{18}$ and THUNDER ${ }^{17}$ trials, there was incomplete outcome data as a few patients did not undergo final angiographic follow-up without a clear explanation (unclear attrition bias).

\section{Discussion}

All available RCTs demonstrate equivalent or favorable technical outcome for DEBs in comparison to standard PTA in the treatment of infrainguinal PAD. The RCTs focused on one of the following primary efficacy end points: LLL, binary restenosis, and freedom from TLR. The follow-up period varied from 6 to 12 months.

Baerlocher et a ${ }^{20}$ recently published a meta-analysis of eight RCTs demonstrating superior results with DEBs over standard PTA for femoral-popliteal disease, as indicated by LLL, restenosis, and TLR. However, no benefit was found in clinical end points, such as major amputation and mortality. In addition, treatment of infrapopliteal disease with DEBs was found to confer improved results compared to standard PTA, as expressed by reduced restenosis and TLR rates.

In terms of clinical end points (wound healing, limb salvage, and mortality), there is no published RCT powered to prove the superiority of DEBs over standard PTA. Several factors can result in improved clinical outcomes, such as a multidisciplinary approach, local wound care, and a surveillance regimen, which may be as important as revascularization.

In terms of new trials currently recruiting patients, the BASIL 3 trial $^{25}$ is a multicenter RCT currently recruiting in 60 UK centers, which aims to determine whether DEB angioplasty with or without bare metal stent, plain balloon angioplasty with or without DESs, or angioplasty with bare metal stent alone is the most effective revascularization 


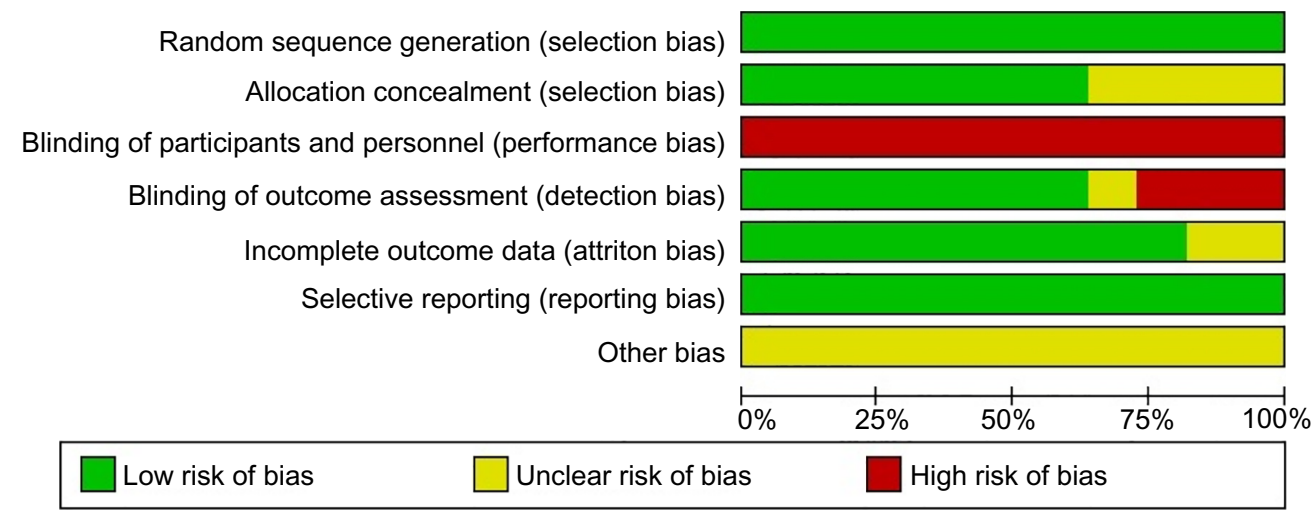

Figure I Risk of bias graph for the studies included in this review.

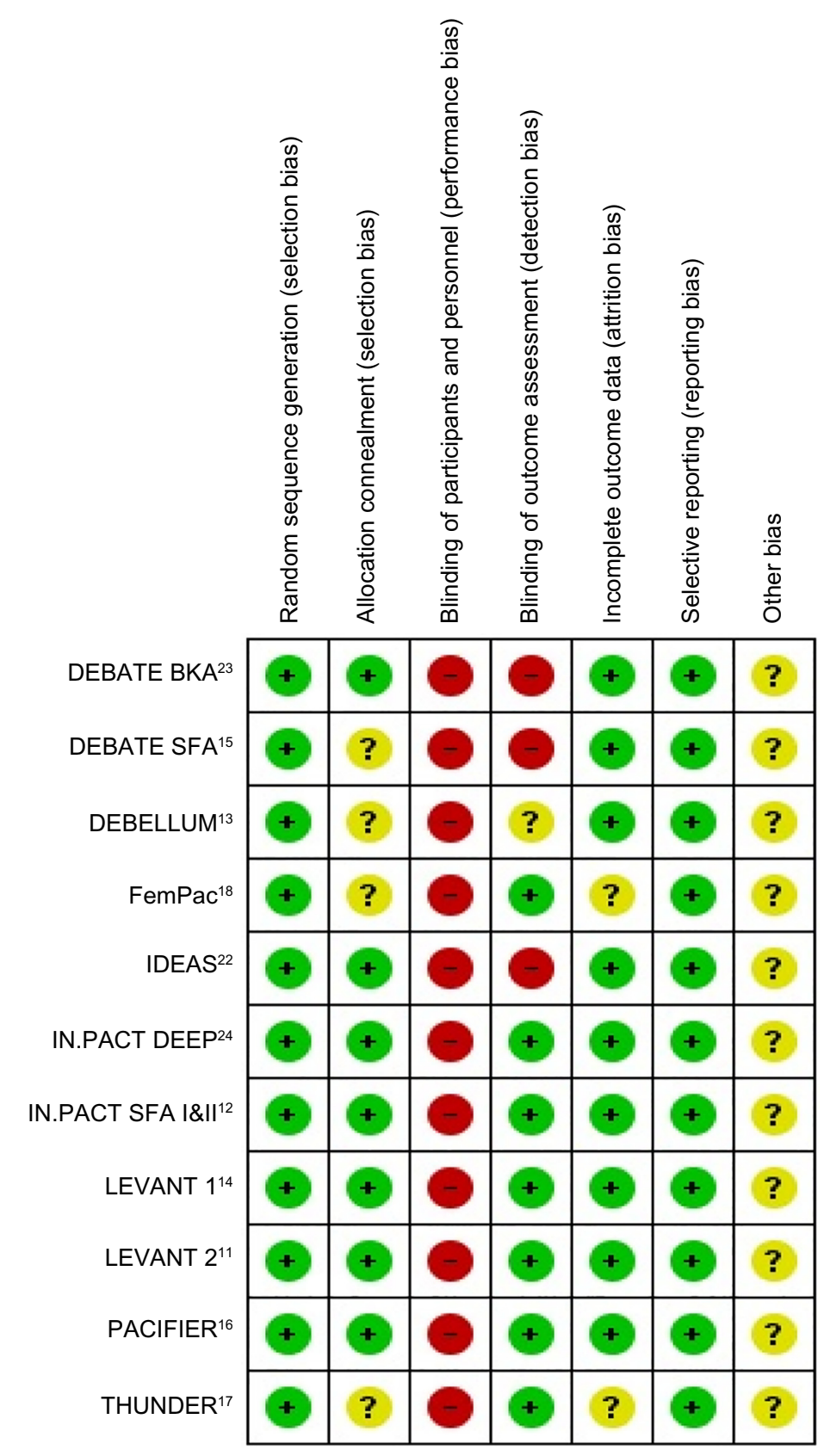

Figure 2 Risk of bias summary: review authors' judgments about each risk of bias item for each included study. Note: +, low risk; ?, unclear risk; -, high risk. 
strategy for severe limb ischemia due to femoral-popliteal disease. Its primary end point is amputation-free survival. In addition, the BASIL 3 trial considers several clinical aspects (ischemic pain relief, ulcer healing, quality of life, 30-day mortality) as secondary outcome measures. The SWEDEPAD trial ${ }^{26}$ is a Swedish RCT testing the hypothesis that DEB is superior to standard PTA in terms of important clinical outcomes, when applied on femoral-popliteal and/or infrapopliteal PAD. The trial consists of two separate parallel studies, SWEDEPAD 1 and SWEDEPAD 2, each defined by the severity of PAD. Patients with CLI are allocated to SWEDEPAD 1 and patients with IC are allocated to SWEDEPAD 2. The primary outcome measures are amputation rate (SWEDEPAD 1) and health-related quality of life (SWEDEPAD 2). The ACOART-BTK trial, ${ }^{27}$ currently recruiting in Italy, is an RCT of DEBs versus standard PTA in the treatment of infrapopliteal disease in patients with CLI. The primary outcome is LLL in the target lesion documented by angiography at 6 months. In Germany, the EffPac trial ${ }^{28}$ is looking at the safety and efficacy of DEBs in inhibiting restenosis and in ensuring long-term patency of superficial femoral artery lesion in comparison to standard PTA. The SINGA-PACLI trial ${ }^{29}$ is another RCT running in Singapore, which is aiming to study the results of DEBs compared to standard PTA for the treatment of infrapopliteal disease in patients with CLI.

Angioplasty with DEBs can have an adverse effect through downstream drug distribution into tissue distal to the lesion location, which may affect wound healing. There are rare cases of vasculitis published in the literature following the use of DEBs.$^{30}$ Furthermore, the endovascular interventionist is potentially exposed to the antiproliferative drug with an unknown long-term risk as all currently used DEBs have the drug coating on top of the balloon.

Even though RCTs have demonstrated technical superiority of DEBs over standard PTA, there are still certain issues to be addressed prior to their widespread use as a primary treatment for patients with PAD. One of the main issues is the lack of a significant difference in major amputation or mortality rates between DEBs and standard PTA. Another issue is the cost implication of DEBs in comparison to standard PTA. Long-term data are still not available from RCTs to support the durability and safety of DEBs.

We noticed a considerable variability in study design, eligibility criteria for patient enrollment, and outcome end points among RCTs. Trials investigating outcomes of DEBs in femoropopliteal disease included mostly patients with IC, whereas patients enrolled in trials investigating treatment of infrapopliteal arterial disease with DEBs had predominantly CLI. One trial examined only diabetic patients with CLI. ${ }^{23}$ The arterial lesions treated with DEBs varied among trials, with some of them treating longer lesions than others. Furthermore, TLR was inconsistently reported among the trials, with some of them reporting clinically driven revascularization.

\section{Conclusion}

DEB provides a novel technique to locally deliver antiproliferative agent into the arterial wall without the need of a chronically implanted delivery system. In PAD, DEB therapy is associated with superior antirestenotic efficacy as compared with standard PTA. DEB angioplasty is a safe procedure. Existing evidence demonstrates no significant differences in major amputation and mortality rate between DEBs and standard balloon angioplasty; however, long-term data are still not available. Further RCTs focusing on the clinical and functional outcomes of DEBs and cost versus clinical benefit are required.

\section{Acknowledgment}

The authors thank Emma Child (Clinical Information Specialist; University Hospital Aintree) for assistance with literature search.

\section{Disclosure}

The authors report no conflicts of interest in this work.

\section{References}

1. Hiatt WR, Hoag S, Hamman RF. Effect of diagnostic criteria on the prevalence of peripheral arterial disease. The San Luis Valley Diabetes Study. Circulation. 1995;91(5):1472-1479.

2. Norgren L, Hiatt WR, Dormandy JA, et al; TASC II Working Group. Inter-society consensus for the management of peripheral arterial disease (TASC II). J Vasc Surg. 2007;45(Suppl S):S5-S67.

3. European Stroke Organisation, Tendera M, Aboyans V, et al; ESC Committee for Practice Guidelines. ESC Guidelines on the diagnosis and treatment of peripheral artery diseases: document covering atherosclerotic disease of extracranial carotid and vertebral, mesenteric, renal, upper and lower extremity arteries: the task force on the diagnosis and treatment of peripheral artery diseases of the European Society of Cardiology (ESC). Eur Heart J. 2011;32(22):2851-2906.

4. Chalmers N, Walker PT, Belli AM, et al. Randomized trial of the SMART stent versus balloon angioplasty in long superficial femoral artery lesions: the SUPER study. Cardiovasc Intervent Radiol. 2013;36(2):353-361.

5. Loh JP, Barbash IM, Waksman R. The current status of drug-coated balloons in percutaneous coronary and peripheral interventions. EuroIntervention. 2013;9(8):979-988.

6. Zeller T, Schmitmeier S, Tepe G, et al. Drug-coated balloons in the lower limb. J Cardiovasc Surg (Torino). 2011;52(2):235-243.

7. Scheller B, Speck U, Abramjuk C, Bernhardt U, Böhm M, Nickenig G. Paclitaxel balloon coating, a novel method for prevention and therapy of restenosis. Circulation. 2004;110(7):810-814.

8. Scheller B. The Invatec IN-PACT Falcon paclitaxel DEB: device description and clinical studies. Presented at: Transcatheter Cardiovascular Therapeutics; September 21-25, 2010; Washington, DC. 
9. Higgins JP, Altman DG, On Behalf of the Cochrane Statistical Methods Group and the Cochrane Bias Methods Group. Cochrane Handbook for Systematic Reviews of Interventions. Version 5.0.1. Chapter 8: Assessing risk of bias in included studies. London: John Wiley and Sons, Ltd; 2008. Available from: http://handbook.cochrane.org/chapter_8/8_assessing_risk_of_bias_in_included_studies.htm. Accessed January 28, 2016.

10. Guyatt GH, Oxman AD, Vist GE, et al; GRADE Working Group. GRADE: an emerging consensus on rating quality of evidence and strength of recommendations. BMJ. 2008;336(7650):924-926.

11. Rosenfield K, Jaff MR, White CJ, et al; LEVANT 2 Investigators. Trial of a paclitaxel-coated balloon for femoropopliteal artery disease. $\mathrm{NEngl}$ J Med. 2015;373(2):145-153.

12. Tepe G, Laird J, Schneider P, et al; IN.PACT SFA Trial Investigators. Drug-coated balloon versus standard percutaneous transluminal angioplasty for the treatment of superficial femoral and popliteal peripheral artery disease: 12-month results from the IN.PACT SFA randomized trial. Circulation. 2015;131(5):495-502.

13. Fanelli F, Cannavale A, Boatta E, et al. Lower limb multilevel treatment with drug-eluting balloons: 6-month results from the DEBELLUM randomized trial. J Endovasc Ther. 2012;19(5):571-580.

14. Scheinert D, Duda S, Zeller T, et al. The LEVANT I (Lutonix paclitaxelcoated balloon for the prevention of femoropopliteal restenosis) trial for femoropopliteal revascularization: first-in-human randomized trial of low-dose drug-coated balloon versus uncoated balloon angioplasty. JACC Cardiovasc Interv. 2014;7(1):10-19.

15. Liistro F, Grotti S, Porto I, et al. Drug-eluting balloon in peripheral intervention for the superficial femoral artery: the DEBATE-SFA randomized trial (drug eluting balloon in peripheral intervention for the superficial femoral artery). JACC Cardiovasc Interv. 2013;6(12):1295-1302.

16. Werk M, Albrecht T, Meyer DR, et al. Paclitaxel-coated balloons reduce restenosis after femoro-popliteal angioplasty: evidence from the randomized PACIFIER trial. Circ Cardiovasc Interv. 2012;5(6):831-840.

17. Tepe G, Zeller T, Albrecht T, et al. Local delivery of paclitaxel to inhibit restenosis during angioplasty of the leg. $N$ Engl J Med. 2008;358(7): 689-699.

18. Werk M, Langner S, Reinkensmeier B, et al. Inhibition of restenosis in femoropopliteal arteries: paclitaxel-coated versus uncoated balloon: femoral paclitaxel randomized pilot trial. Circulation. 2008;118(13):1358-1365.

19. Cassese S, Byrne RA, Ott I, et al. Paclitaxel-coated versus uncoated balloon angioplasty reduces target lesion revascularization in patients with femoropopliteal arterial disease: a meta-analysis of randomized trials. Circ Cardiovasc Interv. 2012;5(4):582-589.
20. Baerlocher MO, Kennedy SA, Rajebi MR, et al. Meta-analysis of drug-eluting balloon angioplasty and drug-eluting stent placement for infrainguinal peripheral arterial disease. J Vasc Interv Radiol. 2015;26(4):459-473.

21. Schmidt A, Piorkowski M, Werner M, et al. First experience with drugeluting balloons in infrapopliteal arteries: restenosis rate and clinical outcome. J Am Coll Cardiol. 2011;58(11):1105-1109.

22. Siablis D, Kitrou PM, Spiliopoulos S, et al. Paclitaxel-coated balloon angioplasty versus drug-eluting stenting for the treatment of infrapopliteal long-segment arterial occlusive disease: the IDEAS randomized controlled trial. JACC Cardiovasc Interv. 2014;7(9):1048-1056.

23. Liistro F, Porto I, Angioli P, et al. Drug-eluting balloon in peripheral intervention for below the knee angioplasty evaluation (DEBATE-BTK): a randomized trial in diabetic patients with critical limb ischemia. Circulation. 2013;128(6):615-621.

24. Zeller T, Baumgartner I, Scheinert D, et al; IN.PACT DEEP Trial Investigators. Drug-eluting balloon versus standard balloon angioplasty for infrapopliteal arterial revascularization in critical limb ischemia: 12-month results from the IN.PACT DEEP randomized trial. J Am Coll Cardiol. 2014;64(15):1568-1576.

25. ISRCTN14469736 [webpage on the Internet]. The BASIL-3 Trial: Balloon vs Stenting in Severe Ischaemia of the Leg-3. Available from: http:// www.isrctn.com/ISRCTN14469736. Accessed January 28, 2016.

26. NCT02051088 [webpage on the Internet]. The SWEDEPAD trial: Swedish Drug-elution Trial in Peripheral Arterial Disease. NCT02051088. Available from: https://clinicaltrials.gov/ct2/show/ NCT02051088. Accessed January 28, 2016.

27. NCT02563535 [webpage on the Internet]. The ACOART-BTK trial: Evaluation of the Use of ACOTEC Drug-Eluting Balloon Litos ${ }^{\circledR}$ in Below-The-Knee Arteries to Treat Critical Limb Ischemia. NCT02563535. Available from: https://clinicaltrials.gov/show/ NCT02563535. Accessed January 28, 2016.

28. NCT02540018 [webpage on the Internet]. The EffPac trial: Effectiveness of Paclitaxel-coated Luminor ${ }^{\circledR}$ Balloon Catheter Versus Uncoated Balloon Catheter in the Arteria Femoralis Superficialis. NCT02540018. Available from: https:/clinicaltrials.gov/show/NCT02540018. Accessed January 28, 2016.

29. NCT02129634 [webpage on the Internet]. The SINGA-PACLI trial: Singapore INfra-Genicular Angioplasty With PAclitaxel-eluting Balloon for Critical Limb Ischaemia. NCT02540018. Available from: https:// clinicaltrials.gov/show/NCT02129634. Accessed January 28, 2016.

30. Thomas SD, McDonald RR, Varcoe RL. Vasculitis resulting from a superficial femoral artery angioplasty with a paclitaxel-eluting balloon. JVasc Surg. 2014;59(2):520-523.
Vascular Health and Risk Management

\section{Publish your work in this journal}

Vascular Health and Risk Management is an international, peerreviewed journal of therapeutics and risk management, focusing on concise rapid reporting of clinical studies on the processes involved in the maintenance of vascular health; the monitoring, prevention and treatment of vascular disease and its sequelae; and the involvement of

\section{Dovepress}

metabolic disorders, particularly diabetes. This journal is indexed on PubMed Central and MedLine. The manuscript management system is completely online and includes a very quick and fair peer-review system, which is all easy to use. Visit http://www.dovepress.com/ testimonials.php to read real quotes from published authors. 